\title{
AVALIAÇÃO DA SEGURANÇA DO PROCESSAMENTO DE FRESAS INTRAMEDULARES FLEXÍVEIS PARA CIRURGIA ORTOPÉDICA
}

\author{
Safety assessment of reprocessing of flexible intramedullary bone reamers for orthopedic surgery \\ Evaluación de la seguridad del procesamiento de fresas intramedulares flexibles para cirugía ortopédica
}

Rafael Queiroz de Souza', Jeane Aparecida Gonzalez Bronzatti², Paulo Roberto Laranjeira', Lycia Mara Jenné Mimica ${ }^{4}$, Cely Barreto da Silva ${ }^{5}$, Aurea Silveira Cruz ${ }^{6}$, Kazuko Uchikawa Graziano ${ }^{7}$

RESUMO: Objetivos: Avaliar a eficácia de um procedimento operacional padrão para limpeza de fresas intramedulares flexíveis, bem como o alcance da esterilidade, e evidenciar a citotoxicidade da sujidade residual de uma fresa flexível utilizada na prática assistencial. Métodos: Fresas intramedulares flexíveis foram pesadas antes do processamento, após contaminação desafio e depois da limpeza. Elas foram contaminadas com Soil Test ${ }^{\mathrm{TM}}$, suspensão de Geobacillus stearothermophilus, na concentração de $10^{6} \mathrm{UFC} / \mathrm{mL}$, e farinha de osso bovino. Após processamento, as amostras foram incubadas em meio de cultura por 21 dias. A sujidade residual de uma fresa utilizada na prática foi submetida ao teste de citotoxicidade in vitro. Resultados: As amostras, embora esterilizadas, apontaram acúmulo de sujidade e o processamento foi ineficaz. A sujidade residual apresentou efeito citotóxico. Conclusão: Recomenda-se que o design flexível das fresas seja descontinuado pela insegurança no processamento. Palavras-chave: Enfermagem. Ortopedia. Esterilização.

ABSTRACT: Objectives: To assess the efficacy of a standard operational procedure to clean flexible intramedullary bone reamers, as well as the sterilization level, and to show the cytotoxicity of the residual dirtiness of a flexible reamer used in care practice. Methods: Flexible intramedullary bone reamers were weighed before processing, after challenge contamination and after cleaning. They were contaminated with the Soil Test ${ }^{\mathrm{TM}}$, Geobacillus stearothermophilus suspension, in the concentration of $10^{6} \mathrm{cfu} / \mathrm{ml}$, and bovine bone flour. After processing, the samples were inoculated into a culture medium and incubated for 21 days. Residual dirtiness of a flexible intramedullary bone reamer used in practice was submitted to in vitro cytotoxicity test. Results: Despite being sterilized, the samples indicate to accumulated dirtiness and the processing was inefficient. Residual dirtiness presented a cytotoxic effect. Conclusion: It is recommended that the flexible design of reamers is discontinued by the lack of safety of reprocessing. Keywords: Nursing. Orthopedics. Sterilization.

RESUMEN: Objetivos: Evaluar la eficacia de un procedimiento operacional estándar para limpieza de fresas intramedulares flexibles, así como el alcance de la esterilidad, y evidenciar la citotoxicidad de la suciedad residual de una fresa flexible utilizada en la práctica asistencial. Métodos: Fresas intramedulares flexibles fueron pesadas antes del procesamiento, tras contaminación desafío y después de la limpieza. Fueron contaminadas con Soil Test ${ }^{\mathrm{TM}}$, suspensión de Geobacillus stearothermophilus, en la concentración de $10^{6} \mathrm{UFC} / \mathrm{mL}$, y harina de hueso bovino. Tras el procesamiento, las muestras fueron incubadas en medio de cultura por 21 días. La suciedad residual de una fresa utilizada en la práctica fue sometida al test de citotoxicidad in vitro. Resultados: Las muestras, aunque esterilizadas, apuntaron acumulación de suciedad y el procesamiento fue ineficaz. La suciedad residual presentó efecto citotóxico. Conclusión: Se recomienda que el design flexible de las fresas sea descontinuado por la inseguridad en el procesamiento.

Palabras clave: Enfermería. Ortopedia. Esterilización.

'Enfermeiro. Doutor em Ciências, Escola de Enfermagem da Universidade de São Paulo - São Paulo (SP), Brasil. E-mail: rafaelqsouza@hotmail.com

${ }^{2}$ Enfermeira. Doutoranda da Escola de Enfermagem da Universidade de São Paulo - São Paulo (SP), Brasil. E-mail: jeanebronzatti@usp.br

${ }^{3}$ Engenheiro Elétrico. Doutorando da Escola de Enfermagem da Universidade de São Paulo -São Paulo (SP), Brasil. E-mail: prlaranjeira@usp.br

4Médica. Professora Doutora, Professora Associada da disciplina de Microbiologia. Departamento de Ciências Patológicas, Faculdade de Ciências Médicas da Santa-Casa de São Paulo - São Paulo (SP), Brasil. E-mail: Imimica@uol.com.br

${ }^{5}$ Farmacêutica bioquímica, Mestre em Ciências, Serviço de Controle de Infecção Hospitalar - Irmandade Santa-Casa de Misericórdia de São Paulo - São Paulo (SP), Brasil. E-mail: cely.silva@santacasasp.org.br ${ }^{6}$ Bióloga. Pesquisadora Científica VI, Doutora pela Universidade de Ciências Farmacêuticas da Universidade de São Paulo, Instituto Adolfo Lutz - São Paulo (SP), Brasil. E-mail: aurcruz@ial.sp.gov.br

'Enfermeira. Professora Titular Sênior do Departamento de Enfermagem Médico-Cirúrgica da Escola de Enfermagem da Universidade de São Paulo e Coordenadora Pedagógica do Curso MBA em Gestão em Centro de Material e Esterilização, Instituto Nacional de Ensino e Pesquisa - São Paulo (SP), Brasil. E-mail: kugrazia@usp.br

Recebido: 27 set. 2016 - Aprovado: 09 dez. 2016

DOI: $10.5327 / 21414-4425201700010004$ 


\section{INTRODUÇÃO}

As fresas ortopédicas intramedulares flexíveis são produtos passíveis de processamento, termorresistentes, de conformação complexa. Elas oferecem dificuldades extremas de limpeza. Esses produtos são constituídos de aço inoxidável e caracterizam-se por uma haste cuja flexibilidade é conferida por duas fitas de aço inoxidável sobrepostas em forma de espiral, uma espiralada no sentido horário e outra no sentido anti-horário, formando uma estrutura flexível semelhante à de uma mola. Essa haste é conectada a uma ponta desenvolvida para desbastar a superfície do canal intramedular de ossos longos.

A dificuldade de limpeza é atribuída não só pela conformação, mas também pela sujidade resultante do próprio procedimento cirúrgico, a qual inclui sangue, osso e medula óssea. A sujidade é distribuída pela haste e grande parte fica retida no espaço criado entre as duas fitas de aço, especialmente nas extremidades, onde a flexibilidade é menor, promovendo grande dificuldade de remoção, pois esse espaço é inacessível aos artefatos e às tecnologias disponíveis para limpeza.

A literatura sobre fresas flexíveis concentra-se em aspectos funcionais e é escassa quanto à segurança na limpeza e na esterilização. Esse fato é preocupante, uma vez que a literatura científica relata sobrevivência de microrganismos na forma vegetativa no instrumental para cirurgias ortopédicas após esterilização pelo vapor, em decorrência de falhas na limpeza.

Em 1999, foi publicado um relato de três casos de artrite séptica por Staphylococcus epidermidis. Os autores identificaram matéria orgânica ressecada em cânulas que seriam utilizadas em procedimentos ortopédicos ${ }^{1}$. Em outro estudo, publicado em 2009, os autores encontraram matéria orgânica em produtos com lúmen, com cultura positiva para Staphylococcus coagulase negativa, Staphylococcus epidermidis e Streptococcus mitis ${ }^{2}$. Em 2011, um surto de Pseudomonas aeruginosa também foi associado a falhas no processamento do instrumental de cirurgia ortopédica, que continha resíduos de matéria orgânica ${ }^{3}$.

Além dos riscos relacionados à infecção associada à dificuldade ou à impossibilidade de limpeza, a toxicidade da sujidade residual contida no interior das fresas ainda não foi evidenciada, e não se sabe o impacto desses resíduos na ocorrência de processos inflamatórios locais e sistêmicos.

Com base nesses relatos, este artigo propôs-se a avaliar a eficácia de um procedimento operacional padrão (POP) para limpeza de fresas intramedulares flexíveis, bem como o alcance da esterilidade, e evidenciar a citotoxicidade da sujidade residual de uma fresa flexível empregada na prática assistencial.

\section{MÉTODOS}

Trata-se de um estudo experimental laboratorial em duas fases, realizado entre 2015 e 2016, e conduzido nos seguintes laboratórios: Laboratório de Ensaios Microbiológicos da Escola de Enfermagem da Universidade de São Paulo, Laboratório de Microbiologia do Departamento de Patologia da Faculdade de Ciências Médicas da Santa-Casa de São Paulo e Núcleo de Cultura de Células do Instituto Adolfo Lutz (São Paulo).

\section{Fase 1: avaliação da limpeza e da esterilidade}

Foram utilizadas como amostra fresas ortopédicas intramedulares flexíveis para úmero, medindo $27,5 \mathrm{~cm}$ de comprimento e $0,4 \mathrm{~mm}$ de diâmetro interno (Tech Tools ${ }^{\circledR}$, Brasil) (Figura 1). Nessa avaliação, usaram-se três fresas recém-fabricadas, sem uso na prática assistencial, identificadas por cores: verde, azul e vermelha.

Para avaliação da limpeza, cada fresa foi pesada em três momentos: antes de cada processamento (peso basal), após contaminação desafio e após limpeza. Então, foi calculado o valor acumulado, dado pela diferença entre peso depois da limpeza e peso basal, utilizando balança com sensibilidade de 0,01 g (Shimadzu Corp., Japão). Para simular o uso assistencial, cada amostra foi submetida à contaminação desafio interna e externamente com Soil Test ${ }^{\mathrm{TM}}$ e suspensão de Geobacillus stearothermophilus, na concentração de $10^{6} \mathrm{UFC} / \mathrm{mL}$, contendo formas esporuladas.

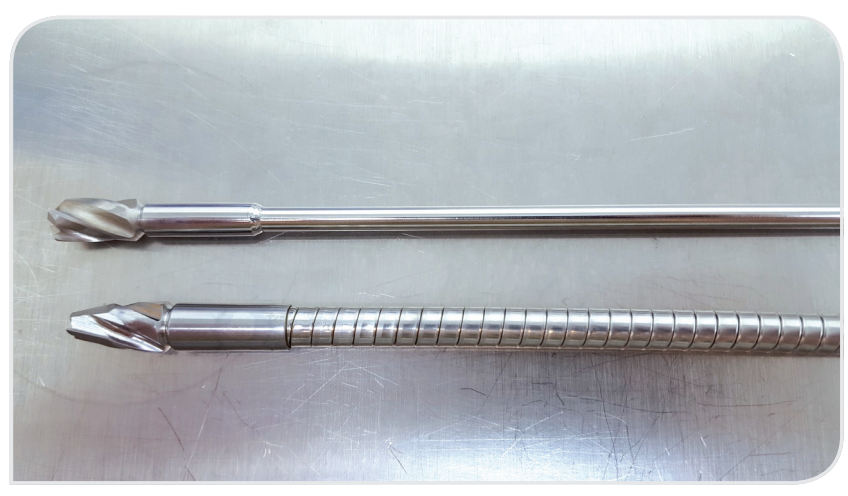

Figura 1. Fresa ortopédica intramedular semirrígida (acima) e fresa intramedular flexível para úmero utilizada como amostra neste estudo (abaixo). 
Após a contaminação com a solução, as amostras foram contaminadas interna e externamente com farinha de osso bovino ( $\sim 3,5 \mathrm{~g})$, simulando os resíduos ósseos ao final de um procedimento cirúrgico ${ }^{4}$. O contato com os contaminantes foi mantido por 3 horas, tempo estimado de um procedimento cirúrgico. Decorrido esse tempo, as amostras foram processadas, de acordo com o POP: pré-umectação em água de torneira por 5 minutos; escovação das superfícies externas da haste (Mack Medical ${ }^{\circledR}$, Brasil); escovação das superfícies externas da ponta (Mack

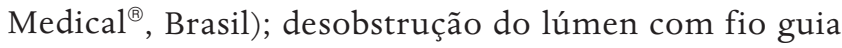
(quando necessário); lavagem do lúmen com pistola de água sob pressão $\left(\mathrm{RFQ}^{\circledR}\right.$, Alemanha) por 5 segundos ou até a desobstrução; escovação do lúmen por cinco vezes

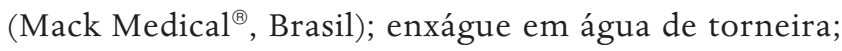
lavagem ultrassônica (Medisafe ${ }^{\circledR}$ SI Digital, Reino Unido) com retrofluxo e detergente enzimático Endozime ${ }^{\mathrm{TM}}$ Xtreme Power (Ruhof ${ }^{\circledast}$, Estados Unidos da América) a $50^{\circ} \mathrm{C}$ por 5 minutos; enxágue com água de torneira; enxágue complementar com água purificada; secagem das superfícies externas com compressa limpa; secagem das superfícies internas com ar comprimido filtrado; inspeção; acondicionamento individual em papel grau cirúrgico/filme (Amcor ${ }^{\circledR}$, Austrália); e esterilização em autoclave a $135^{\circ} \mathrm{C}$ por 4 minutos (Tuttnauer ${ }^{\circledR}$, Israel).

Após a esterilização, as amostras foram inoculadas diretamente em provetas de $250 \mathrm{~mL}$, com meio de cultura caseína soja estéril $\left(\mathrm{BD}^{\circledR}\right.$, Estados Unidos da América). Esse procedimento foi realizado com técnica asséptica, dentro de uma cabine de segurança biológica. Sequencialmente, incubaram-se as provetas a $56^{\circ} \mathrm{C}$ por 21 dias, com leitura diária da recuperação de Geobacillus stearothermophilus.

Esses procedimentos foram repetidos três vezes, de maneira a simular três reúsos de cada amostra. Para atestar a validade dos resultados, os reúsos simulados foram acompanhados com um controle negativo, ou seja, uma fresa nova sem contaminação desafio, submetida ao processamento e incubada em meio caseína soja estéril por 21 dias; e um controle positivo, isto é, uma fresa submetida à contaminação desafio e incubada em meio caseína soja logo depois - ambos a $56^{\circ} \mathrm{C}$.

\section{Fase 2: teste de citotoxicidade in vitro}

Os ensaios de citotoxicidade in vitro são métodos que visam determinar a resposta biológica de células de mamíferos in vitro, por meio de parâmetros biológicos definidos, constituindo uma tentativa de simular ou exagerar as condições de uso clínico, a fim de evidenciar o risco tóxico 5 . Nesta etapa, utilizou-se uma fresa ortopédica intramedular flexível para fêmur após oito reúsos na prática assistencial. A fita de aço externa foi removida e a sujidade aderida à fita interna foi colhida assepticamente em um tubo de ensaio. Após esse procedimento, realizou-se o teste de citotoxicidade in vitro do residual, pelo método da difusão em ágar. Essa análise foi feita em triplicata.

Nesse ensaio, empregou-se a linhagem celular National Collection of Type Cultures (NCTC) clone 929 (célula L, derivada da linhagem L) (American Type Culture Collection ${ }^{\circledR}$ CCL1 $\left.{ }^{\mathrm{TM}}\right)$, catalogada no acervo do Núcleo de Cultura de Células do Instituto Adolfo Lutz sob o número CCIAL020. Para esse teste, os seguintes procedimentos foram adotados: células NCTC clone 929 foram semeadas em placas de Petri tratadas para culturas celulares, com dimensão de $60 \times 15 \mathrm{~mm}\left(\mathrm{TPP}^{\circledR}\right.$, Suíça), em concentração de $3 \times 10^{5}$ células $/ \mathrm{mL}$ e volume de $5 \mathrm{~mL}$; e as culturas foram incubadas por 48 horas, em estufa a $37^{\circ} \mathrm{C} \pm 1^{\circ} \mathrm{C}$, em atmosfera contendo $5 \%$ de $\mathrm{CO}_{2}$. Após esse período, as monocamadas celulares foram avaliadas quanto à confluência, e o meio de cultura substituído por um meio overlay composto de meio de Eagle duas vezes concentrado e ágar ( $\mathrm{BD}^{\circledR}$, Estados Unidos da América) a $1,8 \%$ com $0,01 \%$ de corante vital vermelho neutro. Na preparação ${ }^{6}$, o ágar foi proporcionalmente misturado (1:1) ao meio de Eagle, ambos a $44^{\circ} \mathrm{C}$. A toxicidade celular foi constatada microscopicamente pela alteração da morfologia ou morte das células ao redor ou sob o corpo de prova e macroscopicamente pela formação do halo incolor ao redor do material citotóxico ${ }^{6}$. Depois da medição da extensão do halo incolor feita a partir da amostra, a citotoxicidade foi classificada de acordo com os graus de reatividade para o teste de difusão em ágar descritos na norma ISO 10.993-5:20095.

\section{RESULTADOS}

O POP de limpeza utilizado foi ineficaz, uma vez que não foi capaz de remover completamente a sujidade das amostras. A diferença média de peso nas amostras após os reúsos foi de $0,30 \mathrm{~g}$ (Tabela 1 ).

Embora o POP tenha falhado, o alcance da esterilidade foi demonstrado em todas as amostras, não tendo, portanto, sido observada turvação do meio de cultura ao 
final de 21 dias de incubação. Os resultados dos controles negativo e positivo estiveram conforme o esperado nos três reúsos simulados.

A sujidade residual obtida na fresa intramedular flexível para fêmur apontou efeito citotóxico com grau 3 de reatividade biológica, com média $0,31 \mathrm{~cm}$ de halo nas triplicatas, ou seja, efeito citotóxico moderado.

\section{DISCUSSÃO}

\section{Ineficácia do procedimento operacional padrão}

A legislação brasileira exige que cada etapa do processamento do instrumental cirúrgico e dos produtos para saúde seja realizada por meio de um POP elaborado com base em referencial científico atualizado e normatização pertinente ${ }^{7}$. No presente estudo, mesmo empregando métodos de limpeza manual e automatizada com eficácia demonstrada, a conformação das fresas não permitiu a remoção total da sujidade.

No instrumental de conformação complexa, a impossibilidade da remoção total da sujidade também foi constatada em uma pesquisa realizada no instrumental para cirurgias minimamente invasivas, que utilizou o contaminante ATS $^{\circledast}$ (Artificial Test Soil), composto de $85,2 \mathrm{mg} / \mathrm{mL}$ de proteína, $12,3 \mathrm{mg} / \mathrm{mL}$ de carboidratos e $4,12 \mathrm{mg} / \mathrm{mL}$ de hemoglobina. Nesse caso, os autores obtiveram redução de $99 \%$ nos contaminantes após a lavagem ultrassônica das amostras ${ }^{8}$.

A quantificação de resíduos insolúveis é um método já descrito na literatura para a avaliação da sujidade no instrumental ortopédico. Uma investigação de 2012 utilizou a técnica de filtração em membrana e pesagem da matéria orgânica insolúvel (sangue coagulado e farinha de osso bovinos) em produtos com lúmens para cirurgias ortopédicas?. No presente estudo, essa técnica não foi possível em razão do espaço interno entre as fitas metálicas que constituem o corpo das fresas, que não permitiu a eluição da sujidade insolúvel, sendo, portanto, necessário fazer o controle de resíduos por meio do peso total das amostras.

O mesmo estudo analisou resíduos em dispositivos ortopédicos com lúmen que foram contaminados artificialmente com cimento ósseo e observou retenção de resíduos após dez ciclos de contaminação e limpeza'. Os autores concluíram que a complexidade do design influencia na retenção da sujidade, fato semelhante ao verificado nas fresas intramedulares flexíveis.

Por conta do design da fresa flexível, o POP incluiu várias etapas manuais durante a limpeza, o que demandaria atenção especial dos funcionários da área de limpeza. No decorrer dos experimentos, somente a aplicação das etapas de limpeza, incluindo a secagem, precisou de aproximadamente 15 minutos por amostra.

No cotidiano de um centro de material e esterilização (CME), especialmente em hospitais gerais de grande porte, com grande diversidade no instrumental, seria impraticável que um funcionário investisse 45 minutos de um turno de trabalho de seis horas em apenas três produtos para saúde, uma vez que, além da limpeza, outras atividades também são necessárias, como a recepção, a conferência, a segregação, a desmontagem e o encaminhamento de produtos.

Os POPs, na maioria dos casos, são validados em condições laboratoriais, sem considerar a dinâmica dos processos de trabalho nem o dimensionamento de recursos humanos e infraestrutura. Embora os produtos de conformação complexa exijam POPs mais elaborados, faz-se necessária a observação de que um POP demasiadamente longo e com muitas etapas manuais na limpeza pode predispor ao não seguimento de todas as etapas, sobretudo nos horários de maior demanda na área de limpeza do CME.

Observações não sistematizadas evidenciam que os serviços tendem a sacrificar a eficácia em favor da eficiência dos processos. Em outras palavras, a alta demanda por produção, somada a deficits de infraestrutura e recursos humanos, pode levar à supressão de etapas importantes na limpeza dos produtos. Portanto, é urgente que os fabricantes de produtos para saúde priorizem não só a funcionalidade, como

Tabela 1. Distribuição dos pesos nas fresas ortopédicas flexíveis após os reúsos comparativamente ao peso basal. São Paulo, 2016.

\begin{tabular}{|l|c|c|c|c|c|c|}
\multirow{2}{*}{ Fresa } & \multirow{2}{*}{ Peso basal (g) } & \multicolumn{3}{|c|}{ Peso acumulado (g) } & \multirow{2}{*}{ Peso final (g) } & \multirow{2}{*}{ Diferença (g) } \\
\cline { 3 - 7 } & & 1. Reúso & 2. Reúso & 3. Reúso & \\
\hline Vermelha & 72,58 & 0,28 & 0,31 & 0,18 & 72,88 & 0,30 \\
\hline Azul & 76,17 & 0,31 & 0,30 & 0,23 & 76,52 & 0,35 \\
\hline Verde & 73,27 & 0,31 & 0,23 & 0,19 & Média (g) & 0,24 \\
\hline
\end{tabular}


também o processamento efetivo, investindo em designs mais acessíveis à limpeza manual ou automatizada, como as fresas semirrígidas.

O principal facilitador para a gestão dos POPs no CME é o design acessível para limpeza. A gestão de POPs por marca e tipo de produto é requerida, porém, no caso de um hospital de grande porte que atende a várias especialidades cirúrgicas, haveria um número excessivo de POPs na área de limpeza, o que poderia inviabilizar sua aplicação por razões relacionadas à eficiência dos processos e racionalização do trabalho. Uma alternativa que merece discussão no meio científico é avançar de um POP voltado a cada tipo de produto e marca para a categorização de produtos por design, que teriam um POP standard. A título de exemplo, POP para produtos com lúmen $<5 \mathrm{~mm}$ e POP para pinças cirúrgicas convencionais. Os POPs standard facilitariam a segregação e o direcionamento do fluxo de trabalho na área de limpeza, enquanto os POPs específicos seriam destinados a produtos de conformação exótica ou delicada, como produtos com componentes eletrônicos e cânulas de hidrodissecção para cirurgia oftalmológica.

\section{Alcance da esterilidade nas amostras com sujidade}

Ao contrário do esperado, as amostras, mesmo com sujidade residual, não possibilitaram a recuperação dos microrganismos. Um estudo semelhante contaminou cânulas para reparação de menisco com $0,5 \mathrm{~mL}$ de sangue contendo 200 a 500 UFC de Staphylococcus coagulase negativa, que foram testadas em três POPs:

1. limpeza e enxágue manuais na sala de operação, esterilização no ciclo flash sem embalagem $\left(132^{\circ} \mathrm{C}\right.$ por 10 minutos);

2. limpeza e enxágue com detergente enzimático, jato de água sob pressão, esterilização $\left(132^{\circ} \mathrm{C}\right.$ por 45 minutos);

3. limpeza em lavadora ultrassônica, esterilização $\left(132^{\circ} \mathrm{C}\right.$ por 45 minutos).

Nenhum microrganismo foi recuperado após esses procedimentos, porém os autores ainda observaram sangue nas cânulas processadas nos POPs 1 e $2^{1}$. Esses resultados reforçam a possibilidade de um produto estar sem microrganismos viáveis, mesmo com matéria orgânica aderida, embora não possa ser considerado seguro para uso, em razão da resposta biológica, como síndromes da resposta inflamatória sistêmica e síndromes tóxicas do segmento anterior ocular.

Outra pesquisa evidenciou sujidade grosseira nos produtos canulados do sistema DePuy Mitek ${ }^{\circledR}$ Intrafix, com cultura positiva para Staphylococcus coagulase negativa, S. epidermidis e Streptococcus mitis. Nesse trabalho, os autores identificaram que o CME não tinha escovas com diâmetro adequado para a remoção da sujidade do instrumental ${ }^{2}$, fato que indica um desvio grave no atendimento às boas práticas no processamento de produtos para saúde. Não houve menção ao monitoramento do processo de esterilização pelo vapor, impossibilitando demais interpretações dos resultados obtidos.

Em 2011, durante um surto de infecções associado a falhas no processamento do instrumental de cirurgia ortopédica, os autores apontaram resíduos de matéria orgânica e cerdas de escova nos produtos, além da não adesão às boas práticas, uma vez que cânulas de artroscopia eram lavadas somente com água de torneira. Outro ponto importante é que, mesmo com a observância dos POPs fornecidos pelo fabricante, alguns produtos ainda continham resíduos de matéria orgânica ${ }^{3}$. Nesse estudo, foi notório o fato de que os resíduos foram encontrados em locais cuja visualização só foi possível por meio de um boroscópio. Ou seja, a inspeção visual somente das superfícies externas dos produtos com espaços internos não foi efetiva, reforçando a necessidade do investimento em tecnologias de visualização na área de preparo, monitores de limpeza e qualificação dos equipamentos de limpeza automatizada, conforme exige a legislação brasileira ${ }^{7}$. Adicionalmente, reitera-se a importância de design acessível à limpeza e validação concorrente dos POPs, pois, no caso do estudo supracitado, as instruções do próprio fabricante não foram eficazes.

\section{Residual citotóxico}

A esterilidade é um aspecto crítico da segurança dos produtos para saúde, porém não é o único, uma vez que, mesmo estéril, um produto pode ser tóxico ao organismo. Nos processos de validação de limpeza, a possibilidade de se atingir o "zero absoluto" nos resíduos orgânicos está descartada, embora reduções da ordem de $99 \%$ tenham sido reportadas ${ }^{8}$. Dessa forma, é fundamental que a resposta biológica a essa sujidade residual, mesmo que ínfima, seja demonstrada para que um produto processado seja considerado seguro, conforme proposto por estudos laboratoriais de validação de POPs ${ }^{10,11}$.

Nos dados obtidos, a toxicidade da sujidade residual obteve grau 3. Portanto, risco inaceitável para uso em procedimentos cirúrgicos, salientando a tese de que um produto que não pode ser limpo não pode ser seguramente reutilizado. 


\section{CONCLUSÃO}

O POP utilizado, elaborado na melhor sequência de passos em conformidade com o factível, foi ineficaz; as fresas intramedulares flexíveis não demonstraram recuperação de Geobacillus stearothermophilus, porém a sujidade residual produziu efeito citotóxico. Isso posto, os resultados sustentam a descontinuidade do design flexível pela insegurança no processamento. Ressalta-se que esse fato deve ser considerado pelos comitês de processamento de produtos para saúde, como também por todas as equipes cirúrgicas usuárias, fabricantes de produtos e órgãos reguladores.

\section{AGRADECIMENTOS}

À empresa Tech Tools ${ }^{\circledast}$, que cedeu as amostras utilizadas nos ensaios.

\section{REFERÊNCIAS}

1. Blevins FT, Salgado J, Wascher DC, Koster F. Septic arthritis following arthroscopic meniscus repair: a cluster of three cases. Arthroscopy. 1999;15(1):35-40. doi: 10.1053/ar.1999.v15.015003

2. Parada SA, Grassbaugh JA, Devine JG, Arrington ED. Instrumentationspecific infection after anterior cruciate ligament reconstruction. Sports Health. 2009;1(6):481-5. doi: 10.1177/1941738109347975

3. Tosh PK, Disbot M, Duffy JM, Boom ML, Heseltine G, Srinivasan A, et al. Outbreak of Pseudomonas aeruginosa surgical site infections after arthroscopic procedures: Texas, 2009. Infect Control Hosp Epidemiol. 2011;32(12):1179-86. doi: 10.1086/662712.

4. Haugen SP, Duraiswamy N, Hitchins VM. Quantification by mass of residual debris in reusable medical devices. Biomed Instrum Technol. 2012;Suppl:61-7. doi: 10.2345/0899-8205-12.1.61.

5. International Standard Organization. ISO 10993-5. 2009. Biological evaluation of medical devices part 5: tests for cytotoxicity: in vitro methods.

6. Rogero SO, Lugão AB, Ikeda TI, Cruz AS. Teste in vitro de citotoxicidade: estudo comparativo entre duas metodologias. Materials Research. 2003;6(3):317-20. doi: 10.1590/S1516-14392003000300003
7. Brasil. Ministério da Saúde. Resolução da diretoria colegiada n. ${ }^{\circ} 15$, de 15 de março de 2012. Dispõe sobre requisitos de boas práticas para o processamento de produtos para saúde e dá outras providências. Brasília; 2012

8. Alfa MJ, Nemes R. Manual versus automated methods for cleaning reusable accessory devices used for minimally invasive surgical procedures. J Hosp Infect. 200458(1):50-8. doi: 10.1016/j.jhin.2004.04.025

9. Lucas $A D$, Nagaraja $S$, Gordon EA, Hitchins VM. Evaluating device design and cleanability of orthopedic device models contaminated with a clinically relevant bone test soil. Biomed Instrum Technol. 2015;49(5):354-62. doi: 10.2345/0899-8205-49.5.354.

10. Tamashiro NS, Souza RQ, Gonçalves CR, Ikeda TI, Luz RA, Cruz AS, et al. Cytotoxicity of cannulas for ophthalmic surgery after cleaning and sterilization: evaluation of the use of enzymatic detergent to remove residual ophthalmic viscosurgical device material. J Cataract Refract Surg. 2013;39(6):937-41. doi: 10.1016/j.jcrs.2012.12.039.

11. Souza RQ, Gonçalves CR, Ikeda TI, Cruz AS, Graziano KU. The impact of the final rinse on the cytoxicity of critical products submitted for processing. Rev Esc Enferm USP. 2015;49:87-92. doi: 10.1590/ S0080-623420150000700013. 